\title{
Intelligent User Interfaces in Digital Games for Empowerment and Inclusion
}

\author{
Nicolas Sabouret \\ Univ. Paris-Sud \\ Orsay, France \\ nicolas.sabouret@limsi.fr \\ Erik Marchi \\ TU München \\ Munich, Germany \\ erik.marchi@tum.de
}

\author{
Björn Schuller \\ TU München \\ Munich, Germany \\ schuller@tum.de
}

\author{
Hazaël Jones \\ Montpellier SupAgro, \\ UMR ITAP \\ Montpellier, France \\ hazael.jones@supagro.fr
}

\author{
Lucas Paletta \\ Joanneum Research \\ Graz, Austria \\ lucas.paletta@joanneum.at
}

\author{
Atef Ben Youssef \\ Univ. Paris-Sud \\ Orsay, France \\ atef.ben_youssef@limsi.fr
}

\begin{abstract}
In the context of the development of Serious Games, this position paper sheds light on their possible use to support and enhance social inclusion through the presentation of three research projects in this domain. We first describe the context of our works and research. We then report on several events joining the scientific community that focused on the research on Digital Games for Empowerment and Inclusion. Last, we propose some results of our experience in this domain. The presented work particularly highlights the importance of user-centered design in interdisciplinary cooperation with psychologists, sociologists, vulnerable end users and the practitioners. We stress the relevance of progress beyond the state-of-the-art in recently focused areas of AI, such as, in computational theory-of-mind, in affective multimodal computing, in learning of socio-emotional skills, and in recovery of human attention processes. Typical examples are given to illustrate the projects' research results.
\end{abstract}

\section{Author Keywords}

Serious Games, Intelligent User Interfaces, Digital Games, Empowerment, Inclusion

\section{ACM Classification Keywords}

H.5.m. Information interfaces and presentation (e.g., HCI): Miscellaneous

\section{INTRODUCTION}

The development of Serious Games, i.e., video games technologies that aim to serve other purposes than

This is the author's version of the work. It is posted here for your personal use. Not for redistribution.

/ACE '15,/ November 16 - 19, 2015, Iskandar, Malaysia

Copyright is held by the owner/author(s). Publication rights licensed to ACM.

ACM 978-1-4503-3852-3/15/11 ..\$15.00

DOI: http://dx.doi.org/10.1145/2832932.2832949 entertainment, is the natural result of the positive impacts of such technologies on key societal issues such as public health (e.g., Captain Novolin by Nintendo \& Novo Nordisk; or Staying Alive by Dassault), ecology (e.g., City Rain by Ovolo), well-being (A Decision of Paramount by Thomas Liu; or Mobile Motivator supported by the $\mathrm{AAL}^{1}$ call 4 ). In this context, and with the growing demand for digital tools supporting marginalized people, it seems natural that the research community considers the possible use of the serious game approach for Empowerment and Inclusion. Indeed, in Europe it is estimated today that 120 million people are at risk of social exclusion ${ }^{2}$. Understanding how ICT in general and Digital Games in particular can offer pathways to social inclusion, particularly when used by social inclusion actors, is a key issue for the world's population [7].

A seminal, prepatarory work on this subject was achieved by the Joint Research Centre "Institute for Prospective Technological Studies", commissioned by the Directorate General CNECT, in 2011. An Expert Workshop was held at IPTS in Seville in January 2012 and a report was published in $2013^{3}$. Further to this study, three research projects worked on DGEI (Digital Games for Empowerment and Inclusion) in the framework of the European Union's FP7 programme:

- The ASC-INCLUSION ${ }^{4}$ project $[8,9,10]$ suggested advanced ICT-enabled solutions and serious games for the empowerment of children with Autism Spectrum Conditions (ASC) who are at risk of social exclusion. It combined several state-of-the-art technologies in one comprehensive game environment, including analysis of users' gestures, facial and vocal expressions, training

\footnotetext{
${ }^{1}$ Ambient Assisted Living is a EU project framework

2 http://ec.europa.eu/social/main.jsp?catId=751

${ }^{3}$ J. Stewart et al. The Potential of Digital Games for Empowerment and Social Inclusion of Groups at Risk of Social and Economic Exclusion: Evidence and Opportunity for Policy, EUR Number: 25900 EN, September 2013. http://ftp.jrc.es/EURdoc/JRC78777.pdf

${ }^{4}$ http://asc-inclusion.eu/
} 
through games, text chatting, animation, video and audio clips. Despite the innovative technologies involved, the project was aimed for home use.

- The MASELTOV (Mobile Assistance for Social Inclusion and Empowerment of Immigrants with Persuasive learning technologies and Social Network Services $^{5}$ ) project [6] recognised the major risks for social exclusion of immigrants from the European information society. This project has harnessed the potential of social, situated and opportunistic learning on mobile phones to enhance the social inclusion of immigrants with an intelligent suite of mobile services.

- The TARDIS (Training young Adult's Regulation of emotions and Development of social Interaction Skills ${ }^{6}$ ) project [1], which aimed to build a scenario-based serious-game simulation platform for young people at risk of exclusion, aged of 18-25, in order to explore, practice and improve their social skills.

Empowerment and Inclusion in this paper must be understood in an extended definition as any action that leads people at risk of exclusion, either for social or for medical reasons, to overtake the situation that isolates him/her from the society. We present in this contribution concrete results that show, independently from the application case, how Digital Games can be used to offer a good support for empowerment and inclusion.

\section{DGEI WORKSHOPS}

Three public workshops were organised between 2012 and 2014 to bridge communities and disciplines around the field of DGEI, by inviting respective researchers and experts to discuss their latest perspectives and findings.

In April 2012 in Brussels, Belgium, a first DGEI meeting was limited to members of the three projects (ASCInclusion, MASELTOV and TARDIS). Twelve work-groups were built to study dissemination and exploitation of DGEI results, validation methodologies and indicators, user requirements, engineering and methodology, extension of the community, etc. One of the conclusions was that the current generation of such games increasingly demands for computational intelligence algorithms to help analyse players' behaviour and monitor their motivation and interest to adapt game progress.

In May 2013 in Chania, Crete, the first open workshop on Intelligent DGEI (IDGEI) was organised, in co-location with the Foundation of Digital Gaming conference (FDG 2013) ${ }^{7}$. IDGEI's topics covered, among others aspects of Machine Intelligence in Serious Games, Mobile and Real-World Serious Gaming, Emotion \& Affect in Serious Games, Player behaviour and Attention Modelling, Player-Adaptation and Motivation, Novel Serious Games, and User Studies \& Tests of Serious Games. The goal was to bring forth existing

\footnotetext{
${ }^{5}$ http://www.maseltov.eu

${ }^{6}$ http://tardis-project.eu/
}

efforts and major accomplishments in the design of intelligent serious games in practice, while encouraging the design of novel applications in context of health-oriented gaming, general learning and driving environments, or emergency preparation and to focus on current trends and future directions in the field of Empowerment and Inclusion.

The contributions presented at this workshop can be grouped by three major aspects: social aspects in IDGEI which tackles the questions of social behaviour expression, elicitation and recognition; intelligence of DGEIs, which focuses on developing specific AI algorithms for the application domains, from machine-learning to rule-based systems; and gaming aspects of IDGEI which studies the games-specific dimensions that can foster the use of such systems and improve their impact. These three axes make up for the originality and core difficulty of studying Digital Games for Empowerment and Inclusion. Field experience given in all presentations shows that these three aspects interleave and that they all come to play for the acceptance of DGEIs and for bringing concrete results in terms of inclusion.

After the success of this meeting, a second open workshop on IDGEI was organised in February 2014 in Haifa, Israel, in co-location with the Intelligent User Interface conference (IUI 2014) ${ }^{8}$. The workshop presented the state-of-the-art in the field, focusing particularly on three main thematic aspects: Emotion Recognition and Expression [12, 13], Human Cognition [14] and Novel Game Frameworks [15, 16]. It is interesting to note the evolution from "Intelligence", as it was presented in IDGEI 2013, to "Human Cognition". Indeed, the understanding of the social aspect (supported by emotion recognition and expression), requires not only the Digital Game to show intelligent behaviour but, more precisely, to outline Human-like cognition, i.e., social attitude expression must be based on human-like processes.

This workshop showed that affective computing [11] is a key component in intelligent games for Empowerment and Social Inclusion, since we face questions such as transfer of social cues, extraction and display of motivation and intention for game users, etc. In this context, Intelligent game interfaces must be capable of representing and reasoning about the user's mental state, in order to react appropriately, for example on social cues that inform the system about his/her social attitude or to track the focus and the workload of users in natural mobile interaction. Moreover, novel game frameworks were investigated that focus on upcoming challenges.

A third open workshop was organised in March 2015 in Atlanta, US, again during the Intelligent User Interface

\footnotetext{
${ }^{7}$ http://idgei.fdg2013.org/

8 http://idgei2014.joanneum.at/
} 
conference (IUI 2015) ${ }^{9}$ with a focus on cognitive system aspects of game interfaces and their impact on human behaviour. The contribution showed progress in the three domains of IDGEI: social behaviour recognition and expression, social adaptation and gaming aspects. Several presentations provided concrete results on the impact of training with intelligent digital games in the domain for enhancing social skills. Applications ranged from job interview training to cultural differences leveraging and autistic spectrum disorder. One important conclusion was that the development of such games usually requires expertise from the general gaming domain but, in particular, also technological savoir-faire that is strongly dependant on the application domain, so as to provide intelligent analysis and reactive solutions.

The workshops provided a forum for exchange in experience with intelligent user interfaces and serious games in practice while encouraging the design of novel applications in context of health-oriented gaming, general learning and driving environments, or emergency preparation

\section{EXPERIENCE LEARNT}

Empowerment and Inclusion is a question of utmost importance for the society. However, dealing with this question must be done with great care since any action can have very negative effects. The population we work with, people at risk of exclusion, need special consideration and care. When it comes to using ICT for supporting the inclusion of such a population, one major risk is to rely too much on the system and to assume that it can work without any human vigilance. The work we have achieved in all three projects proves the opposite.

In the TARDIS project [5], our studies showed that there exists a strong complementarity between the objective signal analysis from the computer and the human understanding of the situation by the practitioner. While youngsters in search for employment tend to trust what the machine "tells" them, they fear all sort of unsupervised interaction with such an "intelligent" system. Similar results appear when working with the ASC population [2,9]. The artificial systems greatly support the training, with the capability to replay the same scenes again and again, but the intervention of human practitioners is required for this training in order to bring any concrete result. Figure 1 illustrates the scenario-based serious-game simulation platform that supports social training and coaching in the context of job interviews. This platform enables the creation of a personalised scenario allowing the applicant to exercise and play with the simulation several times with real-condition situations but also with role-based ones. After each session, a debriefing can be organised in order to provide to the user feedbacks on his performance.

When working with inter-cultural differences [3] and migrants' inclusion [6], it is possible to design applications that help people by training them on a classical task they already know about and could achieve in a different cultural context. Here, the role of the domain expert is not directly to

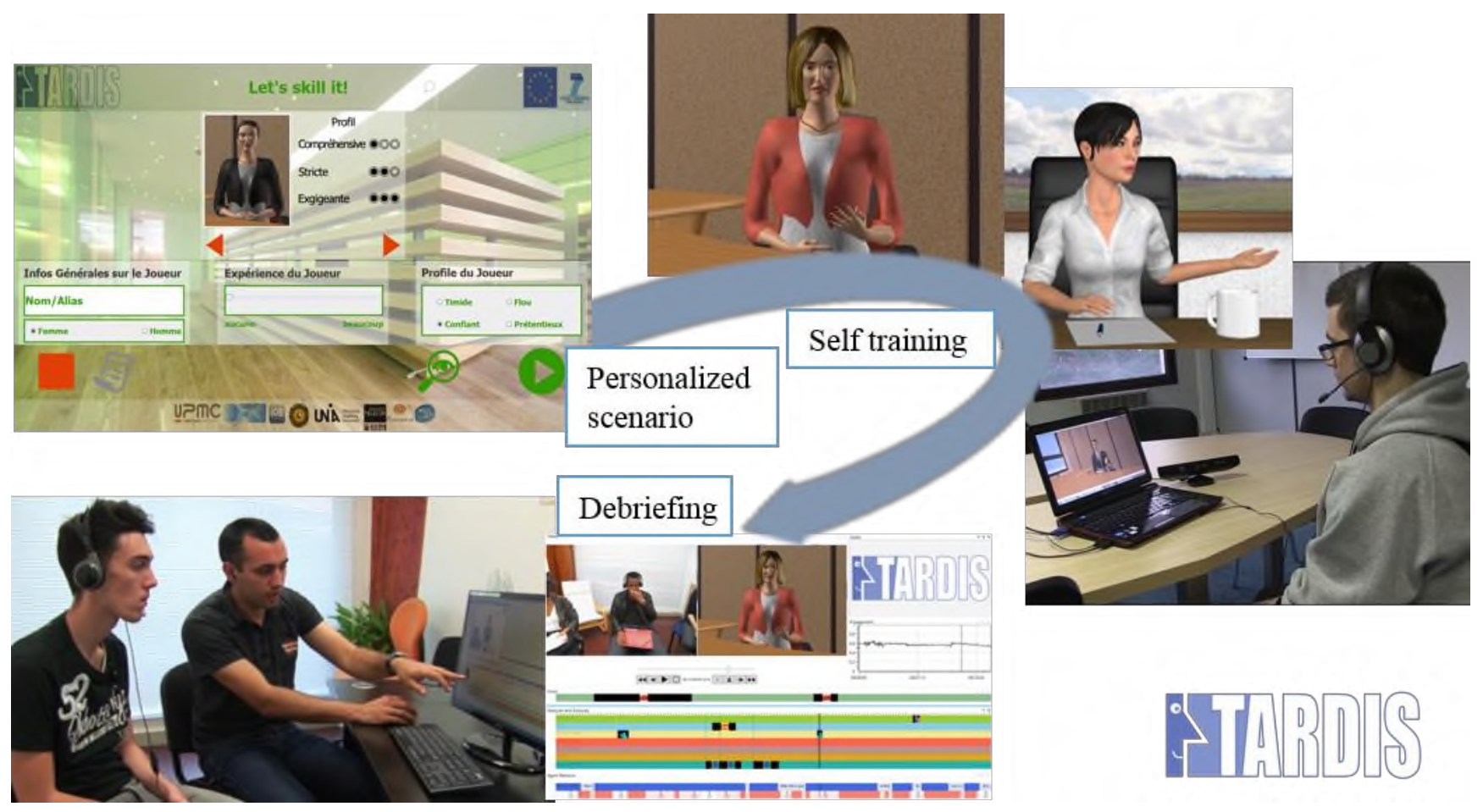

Figure 1. The scenario-based serious-game simulation platform of TARDIS.

\footnotetext{
${ }^{9}$ http://idgei2015.joanneum.at/
} 
intervene during the use of the system but to provide domain knowledge that will make the difference between a standard application and an intelligent, well-suited game for empowerment and inclusion.

Our first conclusion after almost four years of experience in this field is that one must not simply build a DGEI without utmost careful guidance, as this development requires strong collaboration with users, fine-grained studies and as mass approaches (such as large-scale studies with hundreds of users) are not something we can reach at the moment, at least not before practitioners and experts are strongly involved in the use and dissemination of these DGEI.

Our second conclusion refers to the technical development that is required to build Digital Games that can have an impact on the end user's inclusion process. In all application domains we have considered, signal processing and analysis, machine intelligence, and automated adaptation appeared as key features in the game. For example, in social interaction simulations, the system must be able to detect emotions from the user and to provide relevant feedback about it (e.g. [4]). While a lot of research has been done in the field and key results can be shown in well-controlled situations, the results hardly extend to the ecological situation we consider when working with people at risk of exclusion. Developing games with such innovative feature requires reconsidering the Signal Processing and Artificial Intelligence algorithms that we use in classical applications, to take into account the specificities of the population.

\section{EXAMPLES FROM THE PROJECTS}

One illustration of such technical progress driven by the users' needs is the signal processing model in the TARDIS project on job interview simulation for young people Not in Employment, Education or Training (NEETs) [5]. Two difficulties appear as follows.

First, it is not possible to use expensive technology, such as an eye-tracker, or invasive such, such as an ElectroMyoGraphy (EMG) helmet, to obtain hi-fidelity signal quality for affect analysis and recognition. On the contrary, we have to base our algorithms on "simple" signals from a Kinect camera or a regular microphone, with possible noise. However, even in this context, it is important to send feedback to the user, to inform her/him about the perception computed by the system. This requires defining specific algorithms, with specific training, that rely on few signals and that accept a lot of uncertainty.

Second, during the job interviews itself, youngsters hardly show any significant social signals. Their total lack of confidence in their ability to succeed in the interview leads them to a "safe" strategy: they tend to avoid any signal at all, just in case that they could be misinterpreted. In the game, we had to train them to express such social signals - good or bad - in order to receive any feedback from the system. This example illustrates how the AI questions and the game

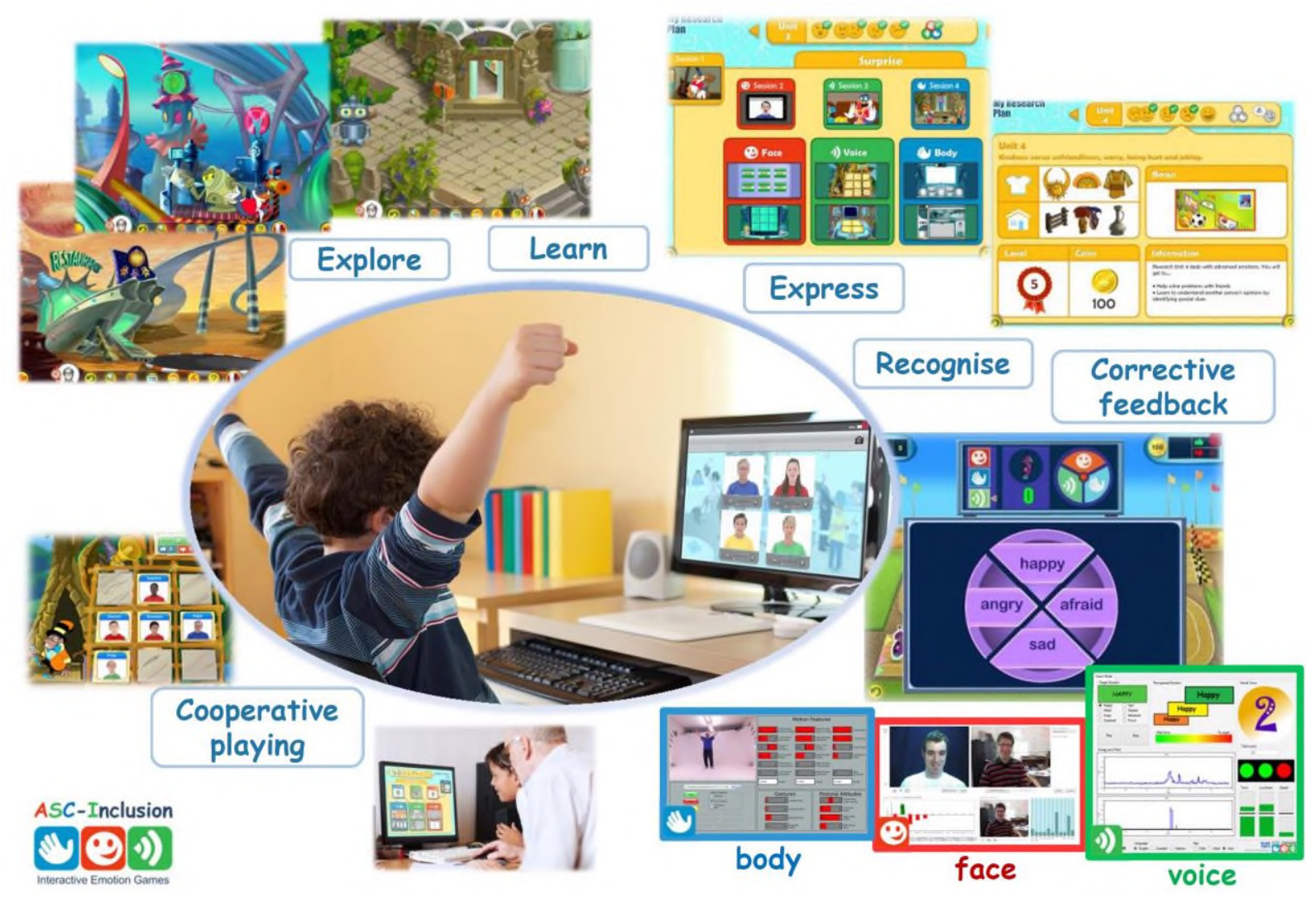

Figure 2. The serious game platform of ASC-Inclusion. 
design interleave and complement each other to build a DGEI that will concretely help people to improve social skills.

In the ASC-Inclusion project, the analysis is performed according to three modalities, namely voice, face, and body gestures for emotion analysis. The most striking aspects which resulted to be relevant were linked to feedback generation. In early stages, all three modalities provided graphical feedback in terms of emotional dimensions such as arousal and valence or emotion categories, however - after pilot trials with users on the spectrum - this form resulted to be difficult and not clear to interpret. Following a collaborative loop between clinicians and technicians, the user interface was refined in order to include an immediate feedback of a user's expressions (e.g., plots tracking user's voice and windows showing the video from the camera with tracking information). This appeared to be engaging and clarifying for the user in the field. Overall, the ASCInclusion platform performs the analysis of users' gestures, facial, and vocal expressions using a standard microphone and webcam for the training through games, animation, video, and audio clips also keeping an eye on low-cost nonintrusive technology. Figure 2 presents the most innovative aspects of the ASC-Inclusion project (from top, clockwise) (i) the child explores the virtual environment visiting different areas such as the research centre where he can (ii) learn about emotions including voice, face and body gesture aspects. The user can then practise his/her learnt skills by (iii) expressing emotions in the three modalities. The ASCInclusion (vi) emotion recognition engine provides (v) corrective feedback based on the child's expression. The ASC-Inclusion platform is also designed to include parents and carers in the game and have them (vi) playing in cooperation with the children. Here, it turned out to be wise to also offer cooperative playing during the course of the project. In the bottom-right part screenshots of the face [10], voice [23], and body gesture [24] analysers are depicted. More details are given in [10].

The importance of how the feedback is provided to the endusers is indeed a relevant aspect and the project also focused on generating formative feedback which produces explanation vectors to measure the difference between the assessed expression and the intended expression. In other words, when children display affective acts, they are not only simply told if their attempt was successful or not, but they also receive a feedback on what they can do concretely to improve their performance. A further example concerns the computational complexity of the analysers. In fact, the ASCInclusion platform is aimed for home use, and processing simultaneously face and body gesture analysis from Kinect and webcam resulted to be heavily demanding for modest domestic computer. This was currently solved by optimising the analysers and by sequencing the processing, albeit using next generation computers may easily solve this issue.
Psychological evaluation of the system took place through an Open Trial (OT). About 20 children in each site went through the intervention. The evaluation from the involved English, Israeli, and Polish sites suggest that the VE is largely liked by users and their parents and perceived as effective in improving children's emotional repertoire. Results show that children with autism improved on emotion recognition and socialization after undergoing the intervention, which suggests that, as a whole, the intervention was effective in helping children learn socio-emotional skills. However, iterative re-design in the loop with the target population was clearly necessary to reach this achievement.

Finally, MASELTOV addresses the specific challenge of providing an intelligent variant of an everywhere/every time service for the purpose of serving the target group of recent immigrants to the European Union by proposing a suite of mobile applications that foster cultural learning and bidirectional integration.

Figure 3 presents the innovative aspects and main pillars of the MASELTOV mobile suite (from top, clockwise): (i) A serious game involves the immigrant user into typical situations and fosters culture understanding - displaying the game shop where players buy equipment based on accumulated coins being gained in successful skill accomplishment, conceptualised as an innovative mixed reality game framework [17]. (ii) Context sensitive services with augmented reality annotation motivate the immigrant player to find useful places they never dared to search for before [20]. (iii) Usability engineering involves a careful analysis of the attention processes based on innovative technologies for large-scale studies recovering eye movements on displays of mobile devices [18]. (iv) Digital logging of behaviour data (displayed is the usage of various MASELTOV components), on- and off-line, enables to investigate aspects of social inclusive smart cities [17,20]. (v) Progress feedback is a highly relevant pedagogical feature of persuasive learning technologies. (vi) Playful mobile language learning (displayed is a list of lesson chapters with subjective and objective performance records) is innovatively embedded in a framework of a context sensitive recommender service $[6,17,20]$

MASELTOV includes a mixed reality game [7,17] in which the user applies her/his language skills in various situations, such as in dialogues during shopping, or for navigation in the urban environment. The mobile service supports her/him in the situation as well as receives feedback from the user in order to measure or estimate performance. The role of gamebased learning in such a context was carefully considered $[6,17]$; the suite of MASELTOV services provides ondemand educational content, and therefore had to be taken to prevent game-based learning services simply presenting a more obtuse representation of this educational content. The serious game also goes beyond its target audience of immigrants, involving and raising awareness amongst the 
general population through the provision of an entertaining game.

The game seeks to apply an experiential learning paradigm, coupled with abstraction and empowerment, to allow the user to experience situations and learn through their actions and responses. Kolb's theory of learning through experience [22] has seen much attention from game- and simulationbased learning communities. It has clear parallels related to how games can allow players to explore problems, devise solutions, and observe the consequences of their actions. The MASELTOV serious game provides a valuable starting point in a novel context - representing a tool for recent immigrant learners - as a proposal to first engage and acquire intrinsic motivation to learn, rather than requiring extrinsic motivators.

To this purpose, we developed a multi-sensory context suite that provides a wealth of behaviour data over time during the interaction of the user with their service and the environment. From the interpretation of these log data the recommendation engine triggers recommender information in real-time that is displayed on the user interface for further notice. In this way, the recommendation engine inter-links and motivates the use of other components in the service suite and reinforces the learning process with continuous persuasive data. This approach enabled to implement sociological or pedagogical research hypotheses into structures of logging mobile gathered spatio-temporal data, in particular for the novel application area of research on immigrant oriented social inclusion $[6,17]$.

In the course of iterative user based evaluation, it became obvious that the acceptance of the user is pivotal to any service provided to her. In this sense, the trust in the service is a prior condition to any utility that the user might take advantage of. Implementing User-Centred Design (UCD) principles, from requirement analysis to participatory interface design and iterative prototype evaluations, appeared as a good solution to build a system that meets the user's needs. Furthermore, MASELTOV applied innovative human factors technologies [18,19] including eye tracking and psycho-physiological sensing in order to investigate in more detail the mechanisms that led to the specific requirements of recent immigrants. While mobile interaction with environments has been studied in lab based context before, MASELTOV developed a usability engineering tool for the recovery and analysis of gaze on mobile displays [19]. This enables investigations of natural behaviour, such as in mixed reality games within task-relevant environments. In a study with 20 test persons (immigrants from Turkey and local natives) involving augmented reality and map based navigation aids for treasure hunt within an Austrian hospital campus, millions of video frames were automatically annotated [18]. The findings drawn from eye tracking data, complementing the qualitative feedback, were capable to reveal that there exist significant differences in the interface

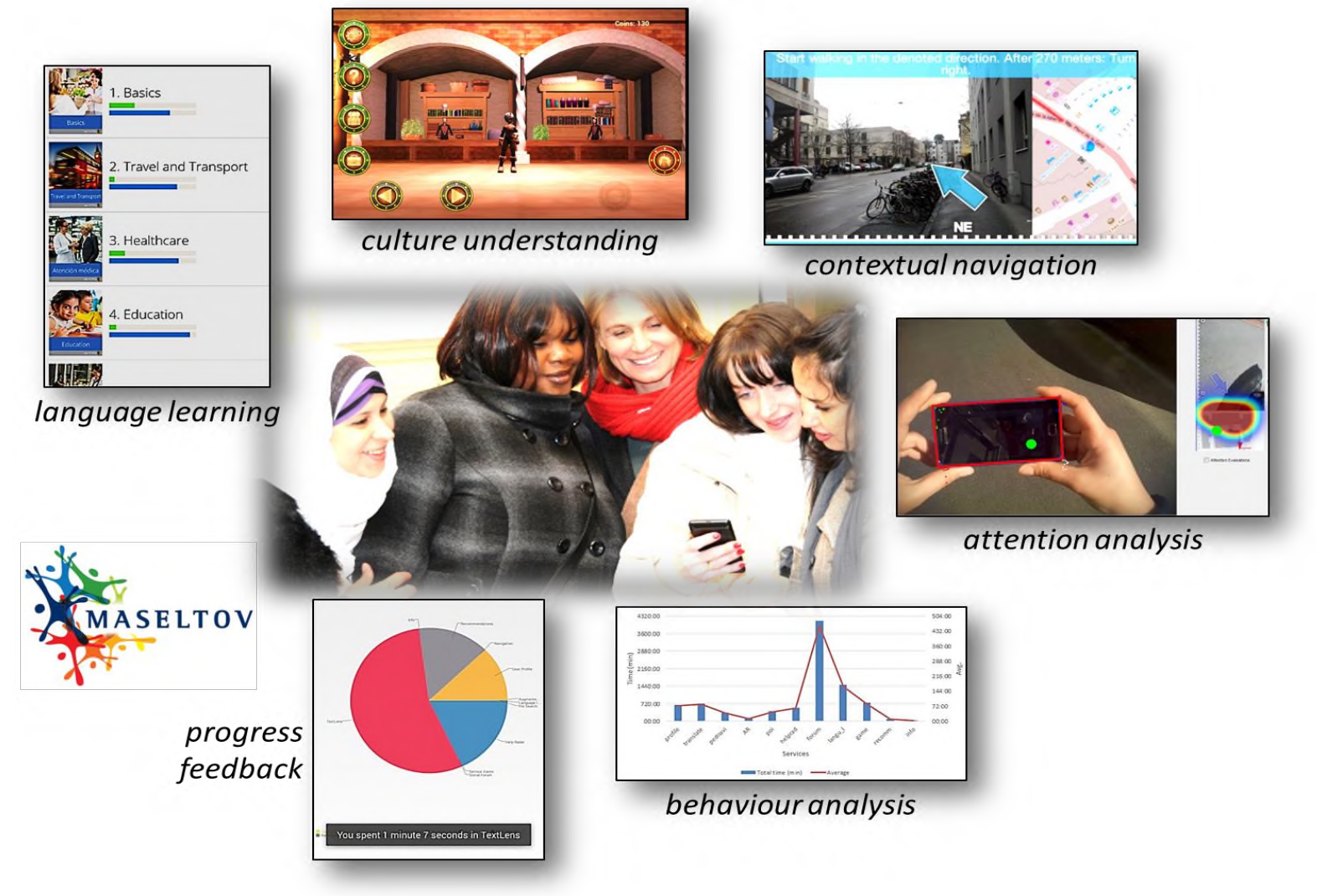

Figure 3. The MASELTOV mobile suite of intelligent services for inclusion of recent immigrants. 
preferences of the Turkish users in contrast to native Austrian behaviours.

We discriminated different usages of selected services from recent immigrants and from local people. The results of these studies clearly show that the interaction behaviour must be explained with psychological models rather than with rational motivations. The uncertainty and insecurity that recent immigrants feel, being exposed to an environment with unknown cultural affordances, makes them either to fully accept or to fully avoid assistance services. Future applications are recommended to gain maximum trust of the user by offering maximum insight and control over the data and functionality.

\section{CONCLUSION}

Digital Games for Empowerment and Inclusion possess the potential to change our society in a positive way by preparing selected groups in a playful and fun way for their everyday life's social and special situations. Exemplary domains span as far as from children with Autism Spectrum Condition to young adults preparing for their first job interviews or migrants familiarising with their new environment. The current generation of such games thereby increasingly demands for computational intelligence in the user interface to help analyse players' behaviour and monitor their motivation and interest to adapt game progress.

Our results show that the impact of these games strongly relies on two considerations. First, practitioners, experts and end-users must be involved in the design process and during the whole use of the system, which makes large-scale dissemination slower, but which brings one to more solid results. Second, on the technical side, building DGEI requires to re-consider the classical Artificial Intelligence and Signal Processing model to take into account the field constraints and the limited technical tools that are accessible to the end-users. Our experience shows that the game incentives can complement with great efficiency the technical limits, when built toward the involvement of the user in the learning process itself. Moreover, it can guide the way the system provides a feedback to the users, which proved, in our three projects, a key question for the acceptance of such a new tool.

Last, the use of user-centred design principles, from requirement analysis to participatory interface design and iterative prototype evaluations, in all three projects, came as a necessary condition for building a successful Digital Game for Empowerment and Inclusion.

Further research in this domain raises both methodological and technical issues. For instance, the use of immersive technology such as the Occulus Rift headset seem as an interesting track to further train people in immersive condition. However, it raises several issues when it comes to using it with fragile populations at risk of exclusions. One of our perspective is to conduct studies to see how such intensive gaming technology impacts the learning outcomes in the context of Empowerment and Inclusion.
From an AI research perspective, the dialogue capabilities of IDGEIs appear as a real bottleneck for the development of more generic solutions that adapt to the user's context. Technology-Enhanced Social Inclusion, and especially Digital Games that are based on precise learning scenario, could benefit from more open dialogues. Part of our current and future research goes toward this direction.

\section{ACKNOWLEDGEMENTS}

The research leading to these results has received funding from the European Community's Seventh Framework Programme (FP7/2007-2013) under grant agreements Nos. 289021 (ASC-Inclusion), 288587 (MASELTOV) and 288578 (TARDIS).

\section{REFERENCES}

1. Anderson, K., André, E., Baur, T., Bernardini, S., Chollet, M., Chryssafidou, E., Damian, I., Ennis, C., Egges, A., Gebhard, P., Jones, Hazaël., Ochs, Magalie., Pelachaud, Catherine., Porayska-Pomsta, K., Paola, Rizzo., Sabouret, N. (2013). The TARDIS framework: intelligent virtual agents for social coaching in job interviews. In Proc. 10th International Conference on Advances in Computer Entertainment Technology (ACE). Twente, the Netherlands, November 12-15, 2013. LNCS 8253.

2. Bernardini, S., Porayska-Pomsta, K., Smith, T. (2014). ECHOES: an Intelligent Serious Game for Fostering Social Communication in Children with Autism. Information Sciences, Elsevier.

3. Degens, N., Hofstede, G.-J., Mascarenhas, S., Silva, A., Paiva, A., Kistler, F., André, E., Swiderska, A., Krumhuber, E., Kappas, A., Hume, C., Hall, L., Aylett, R. (2013). Traveller - Intercultural training with intelligent agents for young adults. In Proc. Intelligent Digital Games for Empowerment and Inclusion (IDGEI), Chaina, Greece, May 14, 2013.

4. Maung, D., Crawfis, R., Gauthier, L., Worthen-Chaudhari, L., Lowes, L., Borstad, A., McPherson, R. (2013). Games for therapy: Defining a grammar and implementation for the recognition of therapeutic gestures. In Proc. Fundations of Digital Games (FDG), Chaina, Greece, May 14-17, 2013.

5. Porayska-Pomsta, K., Rizzo, P., Damian, I., Baur, T., Andre, E., Sabouret, N., Jones, H., Anderson, K., Chryssafidou, E. (2014). Who's Afraid of Job Interviews? Definitely a Question for User Modelling. In Proc. 22nd Conference on User Modeling, Adaptation and Personalization (UMAP), Aalborg, Denmark, July 7-11, 2014.

6. Paletta, L., Dunwell, I., Gaved, M., Bobeth, J., Efremidis, S., Luley, P.-M., Kukulska-Hulme, A., de Freitas, S., Lameras, P., Deutsch, S. (2013). Advances in MASELTOV - Serious Games in a Mobile Ecology of Services for Social Inclusion and Empowerment of Recent Immigrants. In Proc. Advances in Computer Entertainment (ACE), Twente, the Netherlands, November 12-15, 2013. 
7. Schuller, B., Dunwell, I., Weninger, F., Paletta, L. (2013). Serious Gaming for Behavior Change: The State of Play. IEEE Pervasive Computing 12(3): 48-55.

8. Schuller, B., Marchi, E., Baron-Cohen, S., O'Reilly, H., Robinson, P., Davies, I., Golan, O., Friedenson, S., Tal, S., Newman, S., Meir, N., Shillo, R., Camurri, A., Piana, S., Bolte, S., Lundqvist, D., Berggren, S., Baranger, A., and Sullings, N. (2013). ASC-Inclusion: Interactive Emotion Games for Social Inclusion of Children with Autism Spectrum Conditions. In Proc. 1st International Workshop on Intelligent Digital Games for Empowerment and Inclusion (IDGEI 2013) in conjunction with the 8th Foundations of Digital Games 2013 (FDG), ACM, SASDG (Chania, Greece, May 2013).

9. Schuller, B., Marchi, E., Baron-Cohen, S., O’Reilly, H., Pigat, D., Robinson, P., Davies, I., Golan, O., Friedenson, S., Tal, S., Newman, S., Meir, N., Shillo, R., Camurri, A., Piana, Stagliano, A., S., Bolte, S., Lundqvist, D., Berggren, S., Baranger, A., and Sullings, N (2014). The state of play of ASC-Inclusion: An Integrated Internet-Based Environment for Social Inclusion of Children with Autism Spectrum Conditions. In Proc. 2nd International Workshop on Digital Games for Empowerment and Inclusion (IDGEI 2014) held in conjunction with the 19th International Conference on Intelligent User Interfaces (IUI 2014), Computing Research Repository (CoRR), ACM publishing.

10. Schuller, B., Marchi, E., Baron-Cohen, S., Lassalle, A., O'Reilly, H., Pigat, D., Robinson, P., Davies, I., Baltrusaitis, T., Mahmoud, M., Golan, O., Friedenson, S., Tal, S., Newman, S., Meir, N., Shillo, R., Camurri, A., Piana, S., Stagliano, A., Bolte, S., Lundqvist, D., Berggren, S., Baranger, A., Sullings, " N., Sezgin, M., Alyuz, N., Rynkiewicz, A., Ptaszek, K., and Ligmann, K. (2015a). Recent developments and results of ASC-Inclusion: An Integrated Internet-Based Environment for Social Inclusion of Children with Autism Spectrum Conditions. In Proc. 3rd International Workshop on Intelligent Digital Games for Empowerment and Inclusion (IDGEI 2015) as part of the 20th ACM International Conference on Intelligent User Interfaces, IUI 2015, Atlanta, GA. ACM, ACM. 9 pages.

11. Picard, R. Affective Computing, MIT Press, 1998.

12. Cowie, R., Douglas-Cowie, E., Tsapatsoulis, N., Votsis, G., Kollias, S., Fellenz, W., \& Taylor, J. G. (2001). Emotion recognition in human-computer interaction. Signal Processing Magazine, IEEE, 18(1), 32-80.

13. Poggi, I., Pelachaud, C., de Rosis, F., Carofiglio, V., \& De Carolis, B. (2005). Greta. A believable embodied conversational agent. In Multimodal intelligent information presentation (pp. 3-25). Springer Netherlands.

14. Gratch, J., \& Marsella, S. (2004). A domain-independent framework for modeling emotion. Cognitive Systems Research, 5(4), 269-306.
15. Michael, D. R., \& Chen, S. L. (2005). Serious games: Games that educate, train, and inform. Muska \& Lipman/Premier-Trade.

16. Salen, K., \& Zimmerman, E. (2004). Rules of play: Game design fundamentals. MIT press.

17. Dunwell, I., Lameras, P., Stewart, C., Petridis, P., Arnab, S., Hendrix, M., de Freitas, S., Gaved, M., Schuller, B., and Paletta, L.. (2013), Developing a Digital Game to Support Cultural Learning amongst Immigrants, in Schuller, B., Paletta, L., and Sabouret, N., editors. In Proc. 1st International Workshop on Intelligent Digital Games for Empowerment and Inclusion (IDGEI 2013) in conjunction with the 8th Foundations of Digital Games 2013 (FDG), ACM, SASDG (Chania, Greece, May 2013).

18. Paletta, L., Schwarz, S., Bobeth, J., Schwarz, M., and Tscheligi, M. (2015). Gaze Analysis in Mobile Pedestrian Navigation: Culture Affects Wayfinding Styles, Proc. 2nd International Workshop on Solutions for Automatic Gaze Data Analysis (SAGA 2015), in print.

19. Paletta, L., Neuschmied, H., Schwarz, M., Lodron, H., Pszeida, M., Ladstätter, S., Luley, P. (2014), Smartphone Eye Tracking Toolbox: Accurate Gaze Recovery on Mobile Displays, Proceedings of the Symposium on Eye Tracking Research and Applications (ETRA 2014), pp. 367-68, ACM publishing.

20. Gaved, M., Luley, P.M., Efremidis, S., Georgiou, I., Kukulska-Hulme, A., Jones, A.C., Scanlon, E. (2014). Challenges in Context-Aware Mobile Language Learning: The MASELTOV Approach. Proc mLearn 2014, pp. 351364.

21. Dunwell, I., Petridis, P., Lameras, P., Hendrix, M., Doukianou, S., and Gaved, M. (2014). Assessing the Reach and Impact of Game-Based Learning Approaches to Cultural Competency. Proceedings of the 2nd International Workshop on Intelligent Digital Games for Empowerment and Inclusion (IUI2014), arxiv.org, in print.

22. D. A. Kolb and R. Fry, Eds., Toward an applied theory of experiential learning. (Theories of Group Process. London: John Wiley, 1975.

23. Marchi, E., Schuller, B., Baron-Cohen, S., Lassalle, A., O'Reilly, H., Pigat, Golan, O., Friedenson, S., Tal, S., Bolte, S., Lundqvist, D., Berggren, S., and Elfstroem, S.,: "Voice Emotion Games: Language and Emotion in the Voice of Children with Autism Spectrum Condition", Proc. 3rd International Workshop on Digital Games for Empowerment and Inclusion (IDGEI 2015) held in conjunction with the 20th International Conference on Intelligent User Interfaces (IUI 2015), ACM, Atlanta, US, 29.03-01.04.2015.

24. Piana, S., Staglian'o, A., Odone, F., and Camurri, A. Emotional charades. In Proceedings of the 16th International Conference on Multimodal Interaction, ICMI '14, ACM (New York, NY, USA, 2014), 68-69. 